\title{
Reducing the Detrimental Effects of Saturation Phenomena in FRET Microscopy
}

\author{
Tímea Szendi-Szatmári, ${ }^{\dagger}$ Ágnes Szabó, ${ }^{\dagger+\ddagger}$ János Szöllösi, $^{\dagger+}$ and Peter Nagy ${ }^{*}{ }^{\dagger}$ (0) \\ ${ }^{\dagger}$ Department of Biophysics and Cell Biology, Faculty of Medicine, University of Debrecen, Egyetem Square 1, 4032 Debrecen, \\ Hungary \\ ${ }^{\ddagger}$ MTA-DE Cell Biology and Signaling Research Group, Faculty of Medicine, University of Debrecen, Debrecen, Hungary
}

Supporting Information

\begin{abstract}
Although Förster resonance energy transfer (FRET) is one of the most widely used biophysical methods in biology, the effect of high excitation intensity, leading to donor and acceptor saturation, has not been addressed previously. Here, we present a formalism for the experimental determination of the FRET efficiency at high excitation intensity when saturation of both the donor and the acceptor significantly affect conventional FRET calculations. We show that the proposed methodology significantly reduces the dependence of the FRET efficiency on excitation intensity, which otherwise significantly distorts FRET calculations at high excitation intensities commonly used in experiments. The work presented here adds additional rigor to the FRET-based investigation of protein interactions and strengthens the device independence of such results.
\end{abstract}

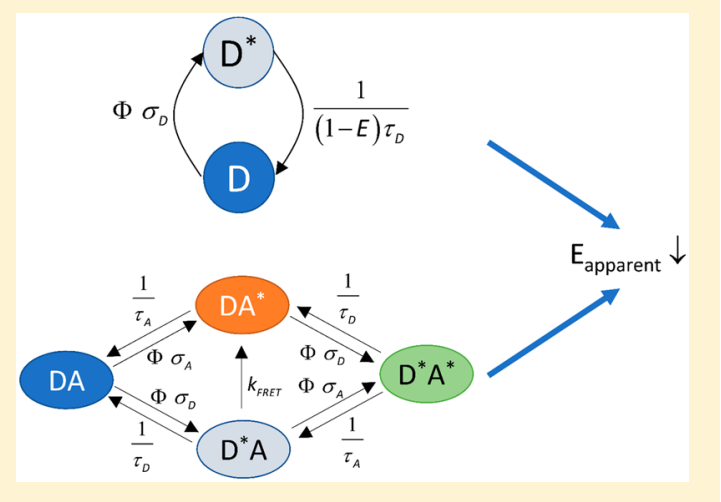

$\mathrm{I}_{\mathrm{p}}^{\mathrm{n}}$ nvestigation of protein interactions under physiological and pathological conditions can shed light on how cells function in health and disease. Förster resonance energy transfer (FRET) has become and still remains a key method for the analysis of protein interactions even in the era of superresolution microscopy owing to its flexibility and relative ease of application. ${ }^{1}$ In FRET, an excited donor transmits energy in a radiationless manner to a suitably oriented acceptor within a couple of nanometers if the absorption spectrum of the latter overlaps significantly with the emission spectrum of the former. This interaction typically leads to donor quenching, shortened donor lifetime, enhanced acceptor emission as well as changes in the anisotropy and photobleaching kinetics of the fluorophores. ${ }^{2}$ Although all of these manifestations lend themselves to different measurement techniques, the most widespread application for measuring FRET is the intensitybased or ratiometric approach in which donor quenching, sensitized and directly excited acceptor fluorescence are measured. ${ }^{3}$ Although a lot of FRET efficiency-related parameters have been introduced, ${ }^{4}$ calculation of the energy transfer efficiency has the charm of being related to the studied interaction in a predictable way due to its solid physical background. ${ }^{5}$ Corrections for photobleaching and for the presence of noncomplexed donors and acceptors have been introduced in order to make the calculations more deviceindependent. ${ }^{6,7}$

The first biological applications of FRET were suggested in the $1960 \mathrm{~s}^{8}$, followed by the development of intensity-based FRET approaches in fluorometry and flow cytometry. ${ }^{9,10}$ Due to the often weak fluorescence signal at physiological expression levels, the investigated targets are commonly overexpressed, leading to mislocalization, ${ }^{11}$ or stained with multiply labeled antibodies, resulting in diminished quantum yield and binding affinity. ${ }^{12}$ Application of strong excitation intensity is also a possible way to increase the signal-to-noise ratio, but fluorophore saturation takes place at commonly applied excitation powers in confocal microscopy. ${ }^{13}$ If fluorophores are saturated, the emitted fluorescence is no longer linearly proportional to the excitation photon flux presenting a major obstacle to standardization. Despite this fact, its effect on intensity-based FRET calculations has not been evaluated. Therefore, the formalism developed for fluorometry and flow cytometry, in which fluorophore saturation is not an issue, has been applied without significant modifications to microscopy. ${ }^{14}$ Although FRET frustration, that is, the absence of FRET if acceptors are saturated, has already been considered, ${ }^{15}$ a complete formalism for intensitybased FRET considering saturation phenomena is not available. As opposed to photobleaching or detector saturation, which are easily identifiable problems related to high excitation intensities, fluorophore saturation is difficult to recognize, and if present, it can distort FRET calculations significantly.

Here, we show that the apparent FRET efficiency calculated according to conventional formulas significantly depends on the excitation photon flux. We present a formalism for evaluating FRET microscopy results taking saturation phenomena into account, and we demonstrate that this approach significantly reduces the dependence of the

Received: March 25, 2019

Accepted: April 17, 2019

Published: April 17, 2019 
calculated FRET efficiency on excitation intensity. The proposed method is crucial for accurate and standardized FRET measurements at commonly applied excitation intensities in microscopy.

\section{MATERIALS AND METHODS}

Cell Line and Antibodies. The human breast-cancer cell line SKBR-3 overexpressing ErbB2 was obtained from the American Type Culture Collection (Rockville, MD) and cultured according to its specifications. For microscopic experiments, cells were grown in eight-well chambered coverglasses (Ibidi, Martinsried, Germany). ErbB2 was labeled by trastuzumab and pertuzumab. Trastuzumab and pertuzumab are humanized monoclonal antibodies against two nonoverlapping epitopes of ErbB2. Trastuzumab was purchased from Roche-Hungary (Budapest, Hungary) and pertuzumab was a kind gift from Genentech (South San Francisco, CA). AlexaFluor488, AlexaFluor546 and AlexaFluor647 dyes (Thermo Fisher Scientific, Waltman, MA) were conjugated to purified monoclonal antibodies according to the manufacturer's specifications.

Labeling of Cells with Antibodies. SKBR-3 cells were grown in 8-well chambered coverglass. Cells were washed twice with ice-cold phosphate-buffered saline (PBS, $\mathrm{pH}$ : 7.4). Cells were labeled with fluorescent antibodies at a concentration of $20 \mu \mathrm{g} / \mathrm{mL}(\sim 130 \mathrm{nM})$ in $150 \mu \mathrm{L}$ of PBS containing $0.1 \%(\mathrm{w} / \mathrm{v})$ BSA on ice in the dark for $30 \mathrm{~min}$. For FRET measurements, cells were labeled with a mixture of donortagged and acceptor-tagged antibodies, while for the determination of parameter $\alpha$ and overspill coefficients, cells were labeled either with donor-conjugated or acceptor-tagged antibodies. In order to remove unbound antibodies the cells were washed twice with PBS followed by fixation in $1 \%$ formaldehyde.

Plasmids and Transfection. In order to measure FRET between fluorescent proteins, cells were transiently transfected with EGFP-mCherry coding for a fusion construct of the two fluorescent proteins separated by a linker (RDPPV). ${ }^{16}$ Spectral overspill factors were determined with cells transfected with pEGFP-C3 (Clontech Laboratories, Mountain View, CA) or with pmCherry-C3 (a kind gift of Julianna Volkó and György Vámosi, University of Debrecen). SKBR-3 cells grown on 8well chambered coverglass were transfected with $0.5 \mu \mathrm{g}$ plasmid/well using Lipofectamin2000 (Thermo Fisher) at a lipid/DNA ratio of 2:1 (v/w) according to the manufacturer's specification.

Confocal Microscopy. A Zeiss LSM 880 confocal laser scanning microscope (Carl Zeiss, Oberkochen, Germany) was used to image the samples. In order to measure FRET between AlexaFluor488-trastuzumab and AlexaFluor546-pertuzumab, excitation of the donor in the donor and FRET channels was performed at $488 \mathrm{~nm}$, and emission was detected in the wavelength range of 500-530 and 550-610 nm, respectively. The acceptor was excited at $543 \mathrm{~nm}$, and its emission was measured between 550 and $610 \mathrm{~nm}$. During FRET measurements between AlexaFluor546-trastuzumab and AlexaFluor647-pertuzumab, AlexaFluor546-trastuzumab was excited by a $543 \mathrm{~nm}$ laser beam, and its emission was detected between 550 and $610 \mathrm{~nm}$ in the donor channel while FRETsensitized fluorescence of the acceptor was measured between 635 and $755 \mathrm{~nm}$. The excitation of AlexaFluor647-pertuzumab was performed at $633 \mathrm{~nm}$, and its emission was detected between 635 and $755 \mathrm{~nm}$. In order to determine FRET in transfected cells the donor (EGFP) was excited at $488 \mathrm{~nm}$ and its emission was measured in the donor channel between 495 and $575 \mathrm{~nm}$ and FRET-sensitized emission of the acceptor was detected between 580-670 $\mathrm{nm}$. The acceptor (mCherry) was excited by a $543 \mathrm{~nm}$ laser line and detected in the wavelength range of $575-695 \mathrm{~nm}$. Fluorescence images were recorded as single optical sections using a $63 \times(\mathrm{NA}=1.4)$ oil immersion objective. A single field of view was measured at four different levels of increasing laser power (1-5-10-15\%), followed by analyzing another field using decreasing excitation powers $(15-10-5-1 \%)$. The measurements were carried out with a pinhole size of 1 Airy unit and a dwell time of $32.97 \mu \mathrm{s}$.

Measurement of Saturation of Mobile Fluorophores. Antibody stock solutions were diluted to $200 \mathrm{nM}$ in PBS in order to measure their intensity. Since fluorophores in solution are mobile, photobleaching is negligible in this case. A relatively large volume $(200 \mu \mathrm{L})$ of this antibody solution was added to a well of an 8-well chambered coverglass in order to prevent unpredictable reflections from the surface of a drop of a smaller volume of solution. Fluorescence intensity was measured as close to the coverglass as possible using excitation and emission settings described in the previous section in two different fields. The excitation intensity was gradually increased in one of the fields (1-5-10-15\% laser power), while it was gradually decreased in the other field (15-10-5-1\%). Since the two measurement types were identical within experimental error, demonstrated in Figure S8, the presented results are averages of both kinds of measurements. The measured fluorescence intensities were normalized to the intensity measured at the lowest excitation power followed by fitting eq S19 in the Supporting Information to these normalized values. The fitting provided the photon flux at the lowest excitation power.

Measurement of Laser Intensity. Laser power measurement was carried out with a Thorlabs (Newton, NJ) optical power meter (PM100D) equipped with a sensor for the spectral range of $350-1100 \mathrm{~nm}$ (S170C). The sensor was placed on the microscope stage after removing the objective. The laser power was measured using continuous illumination in spot scanning mode in order to prevent intermittent exposure of the sensor in raster scanning mode. The intensity of the lasers $(488,543$, and $633 \mathrm{~nm})$ was adjusted on a percent scale on the microscope, and the corresponding laser power was measured with the optical power meter, followed by converting it to photon flux considering the area of the focal spot and the energy of individual photons. ${ }^{17}$

Image Analysis. Image analysis was carried out in Matlab (MathWorks, Natick, MA) supplemented with the DipImage toolbox (Delft University of Technology, Delft, The Netherlands). Membrane pixels were identified with a custom-written implementation of the manually seeded watershed segmentation algorithm. ${ }^{18}$ The FRET efficiency and all the required correction parameters were calculated by rFRET in Matlab ${ }^{19}$ (https://peternagy.webs.com/Matlab/rfret/rfret.zip). The conventional formulas and those taking fluorophore saturation phenomena into account were also entered into an Excel sheet available at the following URL: https://peternagy.webs.com/ Excel/FRET_at_saturation+photon_flux.xlsm.

\section{RESULTS AND DISCUSSION}

At high excitation photon flux FRET-induced quenching of donor fluorescence is mitigated due to the donor being almost instantaneously re-excited after relaxation due to energy 
transfer (Figure 1). A quantitative consideration of this effect, shown in detail in the Supporting Information, predicts that

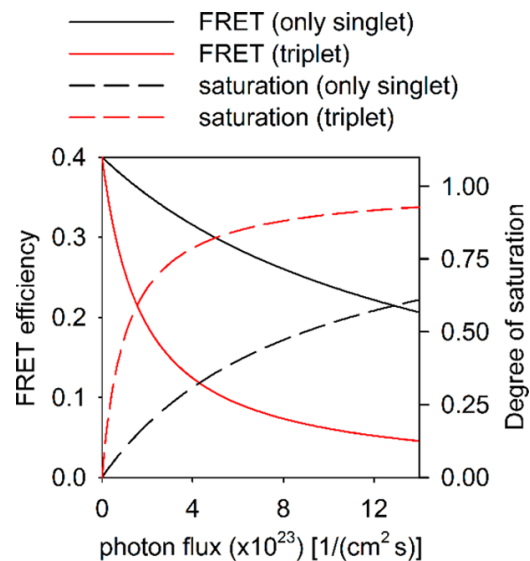

Figure 1. Donor saturation and its effect on the FRET efficiency. Fluorophore saturation in the absence and presence of the triple state was calculated as a function of the excitation photon flux (dashed lines, $\tau=4.1 \mathrm{~ns}, \varepsilon=41000 \mathrm{M}^{-1} \mathrm{~cm}^{-1}, k_{\mathrm{isc}}=7.3 \times 10^{6} \mathrm{~s}^{-1}, k_{\mathrm{ph}}=10^{6}$ $\mathrm{s}^{-1}$ ). The fluorescence lifetime and the molar absorption coefficient are those of AlexaFluor488, while the rate constants were chosen to correspond to a triplet lifetime of $1 \mu \mathrm{s}$ and a triplet quantum yield of 0.03. The fluorophore was assumed to serve as a donor in a FRET interaction characterized by an energy transfer efficiency of 0.4. This system was modeled both in the absence and presence of the triplet state, and the FRET efficiency was calculated from donor quenching using a conventional equation disregarding donor saturation (continuous lines).

the FRET efficiency evaluated from donor quenching $\left(E_{\text {apparent }}\right)$ decreases as a function of fractional donor saturation $\left(D_{\text {sat }}\right)$ :

$$
E_{\text {apparent }}=1-\frac{D_{\mathrm{A}}^{*}}{D_{\mathrm{noA}}^{*}}=\frac{\left(1-D_{\mathrm{sat}}\right) E}{1-D_{\mathrm{sat}} E}
$$

where $E$ is the theoretical FRET efficiency, that is, the fraction of donors relaxing by FRET, $D_{\mathrm{A}}{ }^{*}$ and $D_{\mathrm{noA}}{ }^{*}$ are the concentration of excited donors in the presence and absence of acceptor, respectively. $D_{\text {sat }}$ is the fractional saturation of the donor in the absence of FRET:

$$
D_{\text {sat }}=\frac{\sigma_{\mathrm{D}} \tau_{\mathrm{D}} \Phi_{\mathrm{D}}}{1+\sigma_{\mathrm{D}} \tau_{\mathrm{D}} \Phi_{\mathrm{D}}}
$$

where $\sigma_{\mathrm{D}}$ and $\tau_{\mathrm{D}}$ are the absorption cross-section and fluorescence lifetime of the donor, respectively, and $\Phi_{\mathrm{D}}$ is the excitation photon flux. Rearrangement of eq 1 provides a way to correct the apparent FRET efficiency for donor saturation:

$$
E=\frac{E_{\text {apparent }}}{1+D_{\text {sat }}\left(E_{\text {apparent }}-1\right)}
$$

Since the excitation photon flux commonly used in confocal microscopy is within the range in which such saturation phenomena take place (Figure S1), we concluded that this issue merits further investigation. Equation 1 predicts that the excitation photon flux-dependent decline of $E_{\text {apparent }}$ depends on the theoretical FRET efficiency with small energy transfer values affected to a higher extent (Figure S2). Since as much as $50-80 \%$ of the dye population accumulates in the triplet state depending on fluorophore properties and the excitation power, ${ }^{13}$ intersystem crossing is expected to affect the apparent FRET efficiency as well. As shown in the Supporting Information, the apparent FRET efficiency derived from donor quenching declines as a function of fractional donor saturation, even if the triplet state is populated:

$$
E_{\text {apparent }}=\frac{\left(1-D_{\text {sat }, \mathrm{T}}\right) E}{1-D_{\text {sat }, \mathrm{T}} E}
$$

where $D_{\text {sat, }}$ is the fraction of donors in the excited singlet state normalized to the highest fraction of donors in the $S_{1}$ state in the presence of the triplet state:

$$
D_{\text {sat }, \mathrm{T}}=\frac{S_{1}}{\lim _{\Phi_{\mathrm{D}} \rightarrow \infty} S_{1}}=\frac{D_{\mathrm{sat}}\left(k_{\mathrm{isc}}+k_{\mathrm{ph}}\right)}{D_{\mathrm{sat}} k_{\mathrm{isc}}+k_{\mathrm{ph}}}
$$

where $k_{\text {isc }}$ is the rate constant of intersystem crossing from the $\mathrm{S}_{1}$ to the $\mathrm{T}_{1}$ state, and $k_{\mathrm{ph}}$ is the rate constant of phosphorescence, assumed to be equivalent to the pooled rate constant for relaxation of the $T_{1}$ state. Equation 4 has two important consequences: (i) it is not the fraction of donors in the excited state, but the normalized fractional saturation of the $S_{1}$ state, which determines the apparent decline of the FRET efficiency evaluated from donor quenching; and (ii) if the triplet state is populated, the apparent decrease of the FRET efficiency is even higher (Figure 1).

Since fluorescence is emitted from the $S_{1}$ state, the normalized fractional saturation of the $S_{1}$ state, given by eqs 2 and 5 , can be simply determined by measuring the fractional saturation of fluorescence, enabling us to correct the apparent FRET efficiency for donor saturation. However, two problems must be solved before proceeding to the experimental determination of the FRET efficiency at fluorophore saturation. (i) The equation set taking frustrated FRET into consideration as well (see later) explicitly contains the photon flux; and (ii) $k_{\text {isc }}$ and $k_{\text {ph }}$, required for accurate prediction of the normalized fractional saturation of fluorophores, are difficult to determine. Therefore, instead of measuring the photon flux with a laser power meter, a modified version of eq 2 , shown in the Supporting Information, was fitted to the normalized fluorescence intensity of mobile fluorophores measured at different excitation photon fluxes (Figure S3). If the triplet state is populated, the photon flux determined from this fitting $\left(\Phi_{\text {apparent }}\right)$ will overestimate the real photon flux (eq S20 in the Supporting Information). If the triplet state of the dyes used is populated, but a model disregarding the triplet state (eq 3 ) is used to predict the apparent decrease of the FRET efficiency, substitution of this overestimated apparent photon flux into the model will lead to an accurate prediction of the apparent decrease of the FRET efficiency (eq S21 in the Supporting Information). Consequently, the proposed method only requires the determination of the apparent photon flux, easily available from fluorophore saturation, instead of the complicated measurement of the rate constants of transitions between the singlet and triplet states.

An equation set considering fluorophore saturation was derived in order to eliminate the photon flux-dependence of the calculated FRET efficiency in intensity-based FRET measurements. A parameter, designated by $\alpha$, relating the detectability of excited acceptors to that of excited donors, is also required for intensity-based FRET measurements. Correction for the dependence of $\alpha$ on excitation photon 

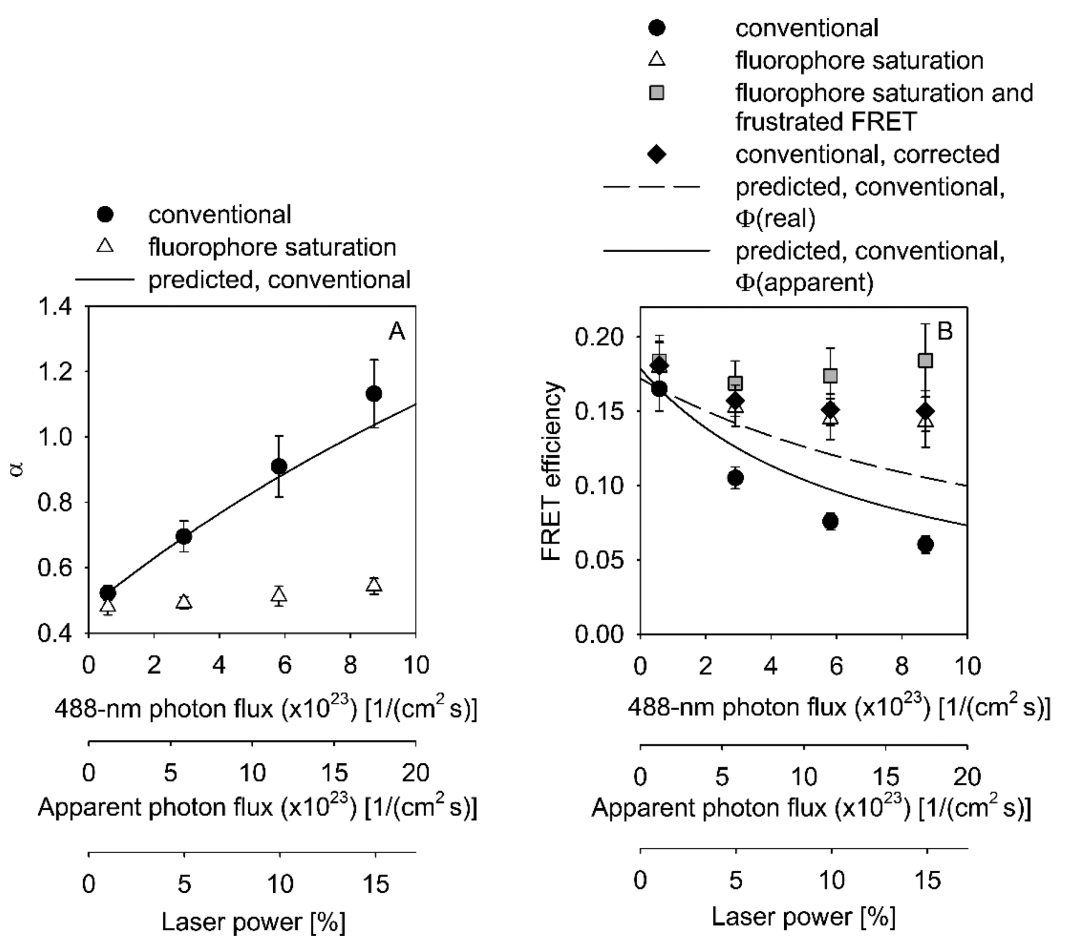

Figure 2. Evaluation of FRET in the AlexaFluor488-AlexaFluor546 donor-acceptor system. (A) SKBR-3 cells were labeled with AlexaFluor488trastuzumab or AlexaFluor546-pertuzumab, and the fluorescence intensities of both the donor- and the acceptor-labeled samples were measured in the donor and FRET channels, respectively, at different intensities of the $488 \mathrm{~nm}$ laser. Parameter $\alpha$ was determined according to the conventional approach, disregarding fluorophore saturation (eq S23 in the Supporting Information) and using the proposed method considering saturation phenomena (eq S24 in the Supporting Information). The continuous line shows the predicted dependence of $\alpha$, calculated in the conventional way, on excitation intensity (eq S26 in the Supporting Information). The real photon flux, the apparent photon flux (eq S20 in the Supporting Information) and the relative intensity on a percent scale, as adjusted on the microscope, are displayed on the horizontal axes in both parts of the figure. (B) Cells were labeled with both AlexaFluor488-trastuzumab and AlexaFluor546-pertuzumab and intensities were measured in the donor, FRET, and acceptor channels at different intensities of the $488 \mathrm{~nm}$ laser. FRET was evaluated in four different ways: conventional disregarding saturation phenomena $(\bullet)$, considering donor saturation $(\triangle)$, considering both donor saturation and FRET frustration (gray $\square$ ), and conventional calculation corrected for donor saturation according to eq $3(\checkmark)$. The dashed and continuous lines show how the FRET efficiency calculated according to the conventional approach is expected to decline as a function of the real photon flux and the apparent photon flux, respectively, according to eq S7 in the Supporting Information. The photon flux of the acceptor-exciting, $543 \mathrm{~nm}$ laser was $9.5 \times 10^{21} 1 /\left(\mathrm{cm}^{2} \mathrm{~s}\right) \mathrm{corresponding}$ to a laser power of $1 \%$.

flux, determination of spectral correction factors, and details of the derivation are described in the Supporting Information. In the calculations discussed so far, frustrated FRET, that is, failure of FRET due to the acceptor being in the excited state, has not been considered. Another set of equations was derived that takes fluorophore saturation and frustrated FRET into consideration (see Supporting Information for details).

The aforementioned principles were used to evaluate FRET in the AlexaFluor488-AlexaFluor546 donor-acceptor pair. Both the FRET efficiency and $\alpha$ declined steeply as a function of the intensity of the donor-exciting laser in accordance with expectations (Figure 2). $\alpha$ corrected for fluorophore saturation was independent of the photon flux. The FRET efficiency was corrected in three different ways: (i) conventional calculation corrected according to eq 3; (ii) considering fluorophore saturation but disregarding frustrated FRET; and (iii) considering fluorophore saturation and frustrated FRET as well. The first two approaches, providing identical results, reduced the photon flux-dependence of the FRET efficiency, while the third one almost completely eliminated it. By comparing results obtained with changing only intensity of donor excitation, only that of acceptor excitation or both, we concluded that the donor excitation photon flux matters the most (Figure S4). These measurements were also carried out with another donor-acceptor pair (AlexaFluor546-AlexaFluor647) leading to identical results (Figure S5).

The approach was also applied to a donor-acceptor pair consisting of two fluorescent proteins $(\mathrm{GFP}+\mathrm{mCherry})$. The equation sets were slightly modified in order to determine both the FRET efficiency and $\alpha$ from the same measurement ${ }^{20}$ (see Supporting Information for details). These measurements also confirmed that the equation sets considering saturation phenomena successfully eliminate the dependence of the FRET efficiency on the excitation photon flux (Figure S6). The estimated and real photon fluxes for GFP were identical in accordance with its very low triplet conversion probability. ${ }^{21}$

Elimination of the strong dependence of the calculated FRET efficiency on excitation photon flux argues for the validity of the underlying assumptions of the proposed method, even though simplifications were introduced for the sake of applicability. From among the multitude of deexcitation pathways available for excited fluorophores, photobleaching and singlet-singlet annihilation, both of which were shown to influence FRET calculations, ${ }^{6,22}$ were neglected. Singlet-singlet annihilation is unlikely to occur significantly in the experimental systems presented in the manuscript for the following reasons: (i) It was shown to eliminate the excitation power-dependence of the apparent FRET efficiency. ${ }^{22}$ The 
mere existence of the excitation photon flux dependence of the calculated FRET efficiency implies that singlet-singlet annihilation does not take place in our systems. (ii) In a complex of one donor and one acceptor singlet-singlet annihilation occurs when both fluorophores are in the excited state $\left(D^{*} A^{*}\right)$. The fraction of such complexes from among all donor-acceptor pairs containing excited donors ( $\mathrm{D}^{*} \mathrm{~A}+$ $\left.D^{*} A^{*}\right)$ was determined according to eq S32. These calculations allowed us to conclude that the population density of $D^{*} A^{*}$ is high only at large FRET values (Figure S7). Since FRET values above $0.3-0.4$ are rarely obtained in cellular FRET measurements, neglecting singlet-singlet annihilation is a reasonable simplifying assumption in such experiments. Photobleaching can also influence FRET measurements by decreasing the amount and density of acceptors and donors and by modifying the donor/acceptor ratio. ${ }^{6}$ Depending on whether the donor and acceptor are randomly distributed or clustered and also on the size of clusters, photobleachinginduced changes in the FRET efficiency range between considerable and negligible. ${ }^{23-26}$ Although dyes underwent photobleaching, resulting in intensity decreases up to $~ 30 \%$ in the three experimental systems investigated in the manuscript, photobleaching did not have a significant effect on the calculated FRET efficiencies, since the FRET values were insensitive to the duration of previous exposure to excitation light (Figure S8). If FRET values calculated in the conventional way or by any of the methods proposed in the current manuscript turn out to be sensitive to bleaching, they should be corrected for bleaching after careful consideration of the applicability of the correction formula.

\section{CONCLUSION}

In conclusion, we derived and applied a formalism for the evaluation of microscopic FRET experiments at photon fluxes leading to fluorophore saturation and frustrated FRET. The proposed method, incorporated into the rFRET Matlab program, ${ }^{19}$ significantly reduces the dependence of the energy transfer efficiency on excitation intensity, which would otherwise distort the measurement. Saturation phenomena must not be overlooked in microscopic FRET measurements in order to add rigor to and increase the device-independence of such experiments.

\section{ASSOCIATED CONTENT}

\section{S Supporting Information}

The Supporting Information is available free of charge on the ACS Publications website at DOI: 10.1021/acs.analchem.9b01504.

Eight figures, Matlab code of functions for evaluating FRET experiments and detailed derivation of the theory of intensity-based FRET calculations in the presence of fluorophore saturation (PDF)

\section{ACKNOWLEDGMENTS}

The work was supported by research grants from the National Research, Development and Innovation Office, Hungary (K120302, GINOP-2.3.2-15-2016-00020, GINOP-2.3.2-152016-00044).

\section{REFERENCES}

(1) Grecco, H. E.; Verveer, P. J. ChemPhysChem 2011, 12, 484-490.

(2) Clegg, R. M. In Laboratory Techniques in Biochemistry and Molecular Biology, Gadella, T. W. J., Ed.; Elsevier, 2009; pp 1-57.

(3) Jares-Erijman, E. A.; Jovin, T. M. Nat. Biotechnol. 2003, 21, $1387-1395$.

(4) Berney, C.; Danuser, G. Biophys. J. 2003, 84, 3992-4010.

(5) Zeug, A.; Woehler, A.; Neher, E.; Ponimaskin, E. G. Biophys. J. 2012, 103, 1821-1827.

(6) Zal, T.; Gascoigne, N. R. Biophys. J. 2004, 86, 3923-3939.

(7) Wlodarczyk, J.; Woehler, A.; Kobe, F.; Ponimaskin, E.; Zeug, A.; Neher, E. Biophys. J. 2008, 94, 986-1000.

(8) Stryer, L.; Haugland, R. P. Proc. Natl. Acad. Sci. U. S. A. 1967, 58, $719-726$

(9) Szöllősi, J.; Trón, L.; Damjanovich, S.; Helliwell, S. H.; ArndtJovin, D.; Jovin, T. M. Cytometry 1984, 5, 210-216.

(10) Trón, L.; Szöllősi, J.; Damjanovich, S.; Helliwell, S. H.; ArndtJovin, D. J.; Jovin, T. M. Biophys. J. 1984, 45, 939-946.

(11) Snapp, E. Curr. Protocols Cell Biol. 2005, 27, 21.4.1-21.4.13.

(12) Szabó, A.; Szendi-Szatmári, T.; Ujlaky-Nagy, L.; Rádi, I.; Vereb, G.; Szöllősi, J.; Nagy, P. Biophys. J. 2018, 114, 688-700.

(13) Tsien, R. Y.; Ernst, L.; Waggoner, A. In Handbook of Biological Confocal Microscopy; Pawley, J. B., Ed.; Springer: New York, 2006; pp $338-352$.

(14) Nagy, P.; Vámosi, G.; Bodnár, A.; Lockett, S. J.; Szöllősi, J. Eur. Biophys. J. 1998, 27, 377-389.

(15) Beutler, M.; Makrogianneli, K.; Vermeij, R. J.; Keppler, M.; Ng, T.; Jovin, T. M.; Heintzmann, R. Eur. Biophys. J. 2008, 38, 69-82.

(16) Renz, M.; Daniels, B. R.; Vámosi, G.; Arias, I. M.; LippincottSchwartz, J. Proc. Natl. Acad. Sci. U. S. A. 2012, 109, E2989-2997.

(17) Wilhelm, S. Confocal Laser Scanning Microscopy; Carl Zeiss: Jena.

(18) Gonzalez, R. C.; Woods, R. E.; Eddins, S. L. In Digital Image Processing Using Matlab, Gonzalez, R. C., Woods, R. E., Eddins, S. L., Eds.; Pearson Prentice Hall: Upper Saddle River, NJ, 2004; pp 417425.

(19) Nagy, P.; Szabó, A.; Váradi, T.; Kovács, T.; Batta, G.; Szöllősi, J. Cytometry, Part A 2016, 89, 376-384.

(20) Szalóki, N.; Doan-Xuan, Q. M.; Szöllôsi, J.; Tóth, K.; Vámosi, G.; Bacsó, Z. Cytometry, Part A 2013, 83, 818-829.

(21) Widengren, J.; Rigler, R. Cell Mol. Biol. (Noisy-le-grand) 1998, $44,857-879$.

(22) Nettels, D.; Haenni, D.; Maillot, S.; Gueye, M.; Barth, A.; Hirschfeld, V.; Hubner, C. G.; Leonard, J.; Schuler, B. Phys. Chem. Chem. Phys. 2015, 17, 32304-32315.

(23) Kenworthy, A. K.; Edidin, M. J. Cell Biol. 1998, 142, 69-84.

(24) Kenworthy, A. K.; Petranova, N.; Edidin, M. Mol. Biol. Cell 2000, 11, 1645-1655.

(25) Hur, K. H.; Macdonald, P. J.; Berk, S.; Angert, C. I.; Chen, Y.; Mueller, J. D. PLoS One 2014, 9, No. e97440.

(26) Yeow, E. K.; Clayton, A. H. Biophys. J. 2007, 92, 3098-3104.

\section{AUTHOR INFORMATION}

\section{Corresponding Author}

*E-mail: nagyp@med.unideb.hu.

\section{ORCID}

Peter Nagy: 0000-0002-7466-805X

\section{Notes}

The authors declare no competing financial interest. 